\title{
Blood flow restriction with different load levels in patients with knee osteoarthritis: protocol of a randomized controlled trial
}

\author{
Roger Andrey Carvalho Jardim ${ }^{1 *}$ D, Tamara Silva de Sousa', Wueyla Nicoly Nascimento dos Santos², \\ Areolino Pena Matos ${ }^{1}$ and Natália Camargo Rodrigues losimuta ${ }^{1}$
}

\begin{abstract}
Background: The effectiveness of blood flow restriction training (BFR) in elderly with knee osteoarthritis (OA) is comparable to performing high-intensity protocols (70 to $80 \%$ of 1 RM [repetition maximum]) that are known to be effective for improving the muscle strength of knee extensors, with the advantage of generating less particular rating of perceived exertion and pain immediately after training. However, despite being a promising alternative, little is known about the best way to apply the BFR, such as level of pressure and combination or not with other therapeutic modalities. The purpose of this study is to evaluate whether different levels of blood flow restriction with low load (BFR + LL) and no load (BFR + rest) are non-inferior to high-intensity resistance exercise (HIRE+BFRplacebo) for pain reduction in patients with knee OA.
\end{abstract}

Methods/design: This clinical trial is a non-inferiority, five-arm, randomized, active-controlled, single trial which will be carried out in 165 patients of both sexes with knee OA, aged 50 years and older. Participants will be randomly allocated into 5 exercise groups (40\% of BFR + LL; $80 \%$ of BFR + LL; $40 \%$ of BFR + rest; $80 \%$ BFR + rest, and HIRE+BFR placebo). A mixed linear model will be used to examine the effect of group-by-time interaction on pain intensity on the WOMAC subscale (primary outcome) and on disease severity, physical functional data, balance data, quality of life, global perceived effect scale, and muscle strength (secondary outcomes). Participants will be analyzed for intention-to-treat, and the statistical assessor blinded to the groups. The collection of outcomes $72 \mathrm{~h}$ after completion of the 16 weeks of interventions will be the primary measurement point. Follow-up secondary timepoints will be collected at 20, 28, 40,52, and 64 weeks after the end of interventions, except for pain during the training, which will be measured immediately at the end of each session. Only the comparison of the primary outcome between the HIRE group with each BFR group will be analyzed in the non-inferiority framework, the other comparisons between the BFR groups for the primary outcome, and all secondary outcomes will be interpreted in the superiority framework.

Discussion: The results of this clinical trial can point out more clearly to ways to optimize the BFR training with the minimum of pain immediately after training, which will allow the offer of an effective and more adherent strengthening training to patients with knee OA.

\footnotetext{
* Correspondence: rogerandcarv@gmail.com

'Postgraduate Program of Health Sciences, Department of Biological

Sciences and Health, Federal University of Amapá - UNIFAP, Macapá, AP, Brazil

Full list of author information is available at the end of the article
}

C The Author(s). 2022 Open Access This article is licensed under a Creative Commons Attribution 4.0 International License, which permits use, sharing, adaptation, distribution and reproduction in any medium or format, as long as you give appropriate credit to the original author(s) and the source, provide a link to the Creative Commons licence, and indicate if changes were made. The images or other third party material in this article are included in the article's Creative Commons licence, unless indicated otherwise in a credit line to the material. If material is not included in the article's Creative Commons licence and your intended use is not permitted by statutory regulation or exceeds the permitted use, you will need to obtain permission directly from the copyright holder. To view a copy of this licence, visit http://creativecommons.org/licenses/by/4.0/ The Creative Commons Public Domain Dedication waiver (http://creativecommons.org/publicdomain/zero/1.0/) applies to the data made available in this article, unless otherwise stated in a credit line to the data. 
Trial registration: Registro Brasileiro de Ensaios Clínicos, RBR-93rx9q. Registered on 23 July 2020. Version 1.0.

Keywords: Osteoarthritis of knee, Blood flow restriction training, Kaatsu training, Muscle strength, Resistance training

\section{Background}

Osteoarthritis (OA) is the most common form of arthritis in the world with an increasing number of cases, resulting from the increase of aging and obesity in the world population [1, 2]. In 2017, there were 307 million people diagnosed with OA in the world [3], resulting in costs ranging from 1 to $2.5 \%$ in the gross domestic product in developed countries [4].

The articular cartilage and subchondral bone of a synovial joint is the main region affected in OA, often resulting in pain, physical disability, and quality of life impairment [5]. Worldwide, the most affected joint in approximately $75 \%$ of all OA cases is the knee joint [1]. The risk factors for the development of knee OA include advanced age, female gender, obesity, previous joint injury, weakness of the knee flexor and extensor muscles, profession, and genetics [6, 7]. Regarding advanced age, the elderly population is especially susceptible to OA because of the loss of global muscle mass [8], gradual reduction of articular cartilage [9], alteration of bone density [10], and reduction in the level of physical activity $[2,11]$.

For the treatment of OA, studies show that strengthening knee extensor muscles is a priority in the management of knee OA [12] with a well-established hypertrophy training protocol for the elderly population [13].

According to current guidelines, physical exercise is recommended to treat knee OA regardless of disease severity, pain levels, and functional status [12, 14-16]. Besides, the recommendations of the World Health Organization's guidelines for physical activity shows that the elderly have to perform at least $150 \mathrm{~min}$ of moderate to vigorous physical activity per week in sessions of at least $10 \mathrm{~min}$. However, only $13 \%$ of older people with knee OA satisfactorily reach that target $[17,18]$. Unfortunately, high-intensity resistance exercise (HIRE) demands the use of high loads that can worsen the pain and cause swelling as well as inflammation in individuals with knee OA [19]. Thus, with the appearance of these symptoms, there is a reduced adherence to exercises [20].

Because of the low adherence of patients with knee OA to HIRE, new training modalities for improving muscle strength have been investigated recently, such as aquatic exercises [21], neuromuscular [22], high speed [23], and blood flow restriction training (BFR) [24].

The BFR has shown promising results in musculoskeletal rehabilitation. It consists of a momentary and controlled mechanical compression of the proximal segment of the limb [25]. The most accepted mechanisms that can explain the development of muscle hypertrophy in BFR is the accumulation of metabolites around the trained muscle as an adaptive response to local hypoxemia [26, 27].

The effectiveness of BFR in patients with knee OA is comparable to traditional protocols for gaining muscle strength in knee muscle extensors [28-30]. However, there is the advantage of generating less joint particular rating of perceived exertion and pain immediately after training [31] and providing results in 6 weeks [32-34] compared to 8 weeks, the training duration commonly needed in HIRE [35].

Despite being a promising alternative, there is still no ideal protocol for applying the BFR in patients with knee OA. Thus, there is still lack of information about the most efficient and safety levels of pressure applied by the cuff to restrict blood flow and on the combination or not of the BFR with other therapeutic modalities [36]. Positive results for muscle strength improvement have been found using the BFR with 40 to $90 \%$ of the total arterial limb occlusion pressure (LOP) [37], associated with resistance exercises using low loads that vary between 10 and $30 \%$ of 1 RM [25]. Yet, no study has assessed whether BFR at rest could also promote similar gains in muscle strength for the knee OA treatment, which theoretically would increase adherence to knee OA treatment by patients, for minimizing the physical discomfort commonly presented in high and moderateintensity exercises [31].

Therefore, the aim of this research is to assess whether different protocols of BFR are non-inferior to HIRE with placebo BFR for pain reduction in patients with knee OA.

\section{Methods/design \\ Study design}

This is a clinical trial of non-inferiority, randomized, active-controlled, single-center trial, with five parallel groups and concealed allocation. Four groups will use BFR with different protocols, and one group will receive HIRE associated with placebo BFR. The intervention groups will be performed for 16 weeks and after this, a follow-up will take place in 6 timepoints, as shown in Fig. 1. The trial was registered at the Registro Brasileiro de Ensaios Clínicos (RBR-93rx9q).

All assessments and the protocol will be carried out in one of the laboratories of the Physiotherapy Course, Federal University of Amapá-AP, Brazil. Participants with knee OA will be recruited from the community in Macapá-AP, Brazil. The invitation will be made through electronic media, folders, personal invitation, and 


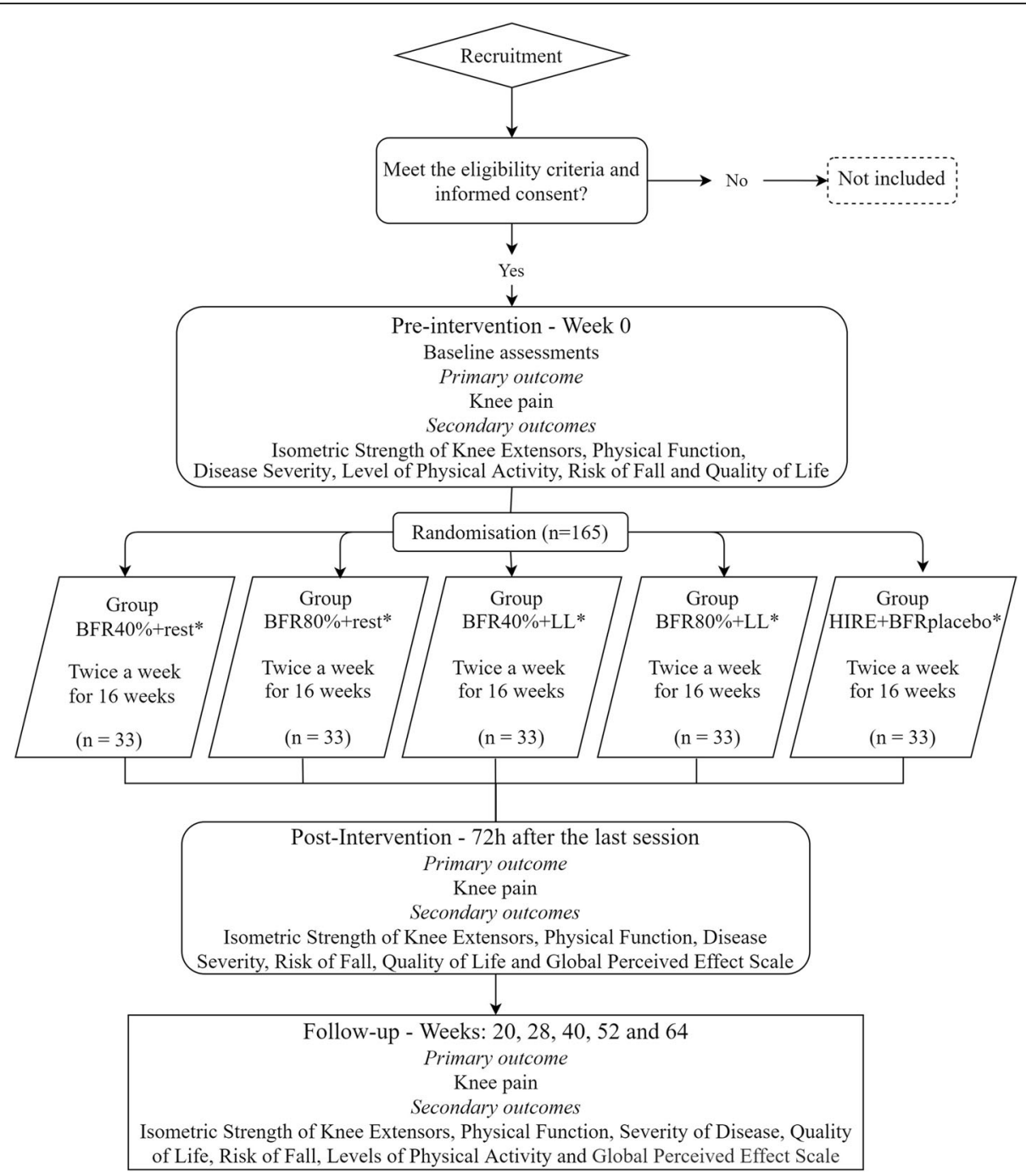

Fig. 1 Consolidated Standard of Reporting Trials flow chart illustrating the process of the study. BFR40\% + rest: restriction of $40 \%$ of blood flow without load. BFR80\% + rest: 80\% restriction of blood flow without load. BFR40\% + LL: 40\% restriction of blood flow with low load. BFR80\% + LL: $80 \%$ restriction of blood flow with low load. HIRE+BFRplacebo: high-intensity resistance exercise with $80 \%$ of a repetition maximum and blood flow restriction placebo. *Immediately upon the conclusion of the sessions, the results of the knee pain will be recorded

telephone contact. Outcome measures will be performed according to the sequence and schedule shown in Fig. 2.

The study protocol was developed according to the guidelines of the standard items of the protocol: Recommendations for interventional trials (SPIRIT) guidelines (Additional file 1) [38] and Consolidated Standards of Reporting Trials (CONSORT) guidelines [39].

\section{Eligibility criteria}

In total, 165 participants will be included if:

1. Over 50 years old;

2. Diagnosed with bilateral or unilateral knee OA according to the American College of Rheumatology criteria;
3. Moderate to very severe (scores between 5 and 13 on the Lequesne Questionnaire);

4. Minimum score of 24 points on the Mini-Mental State Examination and those who sign the Informed Consent Form (ICF).

Patients will be excluded if they have:

1. History of surgery or any invasive procedure on the knee(s) with OA;

2. Undergone a physical therapy or muscle strengthening program for lower limb in the past 3 months;

3. Planned events, such as knee replacement or travel, which may interfere with the adherence of individuals during the trial period; 


\begin{tabular}{|c|c|c|c|c|c|c|}
\hline \multirow[b]{3}{*}{ TIMEPOINT } & \multicolumn{6}{|c|}{ STUDY PERIOD } \\
\hline & \multirow{2}{*}{$\begin{array}{c}\text { Screening } \\
\text { Week }-1\end{array}$} & \multirow{2}{*}{$\begin{array}{c}\text { Baseline } \\
\text { Week } 0\end{array}$} & \multicolumn{2}{|c|}{ Sixteen weeks } & \multicolumn{2}{|c|}{ Follow-up } \\
\hline & & & Odd weeks & Even weeks & $72 h$ & $\begin{array}{l}\text { Weeks 20, 28, } 40 \\
52 \text { and } 64\end{array}$ \\
\hline \multicolumn{7}{|l|}{ ENROLMENT: } \\
\hline \multirow{2}{*}{$\begin{array}{l}\text { Eligibility screen } \\
\text { Informed consent }\end{array}$} & $\mathrm{X}$ & & & & & \\
\hline & $\mathrm{X}$ & & & & & \\
\hline Allocation & & $\mathrm{X}$ & & & & \\
\hline \multicolumn{7}{|l|}{ INTERVENTIONS: } \\
\hline \multicolumn{7}{|l|}{ BFR $40 \%+$ rest } \\
\hline \multicolumn{7}{|l|}{ BFR $80 \%+$ rest } \\
\hline \multicolumn{7}{|l|}{$B F R 40 \%+L L$} \\
\hline \multicolumn{7}{|l|}{$B R F 80 \%+L L$} \\
\hline \multicolumn{7}{|l|}{ HIRE+BFRplacebo } \\
\hline \multicolumn{7}{|l|}{ ASSESSMENTS: } \\
\hline $\begin{array}{r}\text { WOMAC (Likert } \\
\text { scale) }\end{array}$ & & $\mathrm{X}$ & & & $\mathrm{X}$ & $\mathrm{X}$ \\
\hline Lequesne & & $\mathrm{X}$ & & & $\mathrm{X}$ & $\mathrm{X}$ \\
\hline Functional test & & $\mathrm{x}$ & & & $\mathrm{X}$ & $\mathrm{X}$ \\
\hline \multirow{2}{*}{$\begin{array}{r}\text { Risk of falls } \\
\text { Isometric strength of } \\
\text { knee extensors }\end{array}$} & & $\mathrm{X}$ & & & $\mathrm{X}$ & $\mathrm{X}$ \\
\hline & & $\mathrm{X}$ & & & $\mathrm{X}$ & $\mathrm{X}$ \\
\hline$S F-36$ & & $\mathrm{X}$ & & & $\mathrm{X}$ & $\mathrm{X}$ \\
\hline MMSE & & $\mathrm{X}$ & & & & \\
\hline IPAQ & & $\mathrm{x}$ & & & & $\mathrm{x}$ \\
\hline TOP & & $\mathrm{X}$ & & $\mathrm{X}$ & $\mathrm{X}$ & \\
\hline 7-10 RM test & & $\mathrm{X}$ & & $\mathrm{X}$ & $\mathrm{X}$ & \\
\hline VAS during exercise & & & $\mathrm{x}$ & $\mathrm{X}$ & & \\
\hline GPE & & & & & $\mathrm{X}$ & $\mathrm{X}$ \\
\hline
\end{tabular}

Fig. 2 Study design schedule in accordance with the Standard Protocol Items: Recommendations for Interventional Trials. BFR40\% + rest: restriction of $40 \%$ of blood flow without load. BFR80\% + rest: $80 \%$ restriction of blood flow without load. BFR40\% + LL: $40 \%$ restriction of blood flow with low load. BFR80\% + LL: 80\% restriction of blood flow with low load. HIRE+BFRplacebo: high-intensity resistance exercise with $80 \%$ of a repetition maximum and blood flow restriction placebo.WOMAC: Western Ontario and McMaster Universities. SF 36: Short-Form Health Survey. MMSE: Mini-Mental State Exam. IPAQ: International Physical Activity Questionnaire. TOP: Total Arterial Limb Occlusive Pressure. RM: Repetition Maximum. VAS: visual analog scale for pain. GPE: Global Perceived Effect

4. History of acute myocardial infarction and/or stroke;

5. History of peripheral arterial disease or deep vein thrombosis;

6. History of cancer that has generated limitations or restrictions to physical exercise;

7. Decompensated systemic blood pressure without medical supervision;
8. Changes in the dose or type of anti-inflammatory or analgesic drugs in the last 3 months.

One of the researchers (WNNS) will check the volunteers' eligibility criteria and apply the study ICF. Participants who discontinue their attendance in the study will be invited to participate in the assessments during the follow-up period (Fig. 2). 
Thus, all individuals will be included in the analysis as for their intention to treat. Participants who discontinue the proposed treatment and the assessments will be considered as subject loss to follow-up, which should not exceed $15 \%$ of the original sample size. The therapists who will be responsible for the intervention groups will receive specific training for the treatment protocol.

\section{Randomization}

Participants will be randomly allocated in the equal ratio between groups $(1: 1: 1: 1: 1)$ using the generator available at www.randomization.com by a researcher (APM) who will not be aware of the research group participants and evaluations. Balanced permutations in blocks will be used in relation to the presence of unilateral or bilateral knee OA.

The allocation of participants will be hidden in opaque numbered and sequentially sealed envelopes, prepared before the study by a researcher (APM) who did not participate in the recruitment and assignment of the groups. The corresponding envelopes will be opened once the participants enrolled have completed all baseline assessments.

The researcher responsible for evaluations (TSS) and data analysis (NCRI) will be blind to the groups of participants. The order of the evaluations will follow a predetermined sequence that will try to reduce the interference of the physical fatigue of a test in its successor:

- Set 1: WOMAC, MMSE, and LOP;

- Set 2: IPAQ, Time Get Up and Go Test (TUG) and 30-second Chair Stand Test (30-sCST);

- Set 3: SF-36, Lequesne and isometric strength evaluation of the knee extensors;

- Set 4: Clinical Test of Sensory Interaction and Modified Balance, 40-m Fast Paced Walk Test (40mFPWT), Demographic Data and 7-10 RM test.

\section{Intervention}

The intervention protocol will take place in one of the laboratories of the Physiotherapy Course, Federal University of Amapá-AP. The researcher (RACJ), who is a physiotherapist and specialized in BFR and HIRE techniques, will be responsible for the intervention groups.

In a systematic review, Borde, Hortobágyi, and Granacher [13] identified the main parameters of resistance training capable of increasing muscle strength in the elderly. In accordance with this finding, the interventions will take place twice a week with a 40-min duration, for 16 weeks, totalizing 32 sessions (Fig. 2). The reference for the volume of exercises will be provided by the HIRE group, which will perform 3 sets of 8 repetitions at $80 \%$ of $1 \mathrm{RM}$ load, with $60 \mathrm{~s}$ of rest between sets [13]. The total volume of the exercises will be calculated by multiplying the weight, repetitions, and series [19]. For groups with restricted blood flow and low load with $40 \%$ of LOP (BFR40\% + LL) and with $80 \%$ of occlusion $($ BFR $80 \%+\mathrm{LL})$, the load will be fixed at $30 \%$ of $1 \mathrm{RM}$ [40], and the maximum number of repetitions will be 15 repetitions per series, increasing the serial number according to the volume equivalence used in the HIRE group.

For groups with BFR without load with $40 \%$ of LOP $($ BFR $40 \%+$ rest) and with $80 \%$ of occlusion (BFR80\% + rest), the reference for the restriction time will be the average restriction time of the groups with low load, with a minimum of $10 \mathrm{~min}$ per session [24]. The choice for BFR pressures is based on studies that have demonstrated a significant decrease in blood flow from $40 \%$ of the LOP [41], with no additional improvement until the relative pressure of $80 \%$ [42]. The summary of interventions is compiled in Table 1.

The $30 \%$ loads of $1 \mathrm{RM}$ for the BFR $40 \%+\mathrm{LL}$ and BFR $80 \%$ + LL groups, and $80 \%$ for the HIRE+BFRplacebo group, will be estimated (1 RM estimate) from the maximum load that can be exceeded in 7 to 10 repetitions (7 to $10 \mathrm{RM}$ test) [43], based on the Brzycki equation $(W /(1.0278-0.0278 \times R)$, where $W$ refers to the weight used in repetitions until failure and $R$ refers to repetitions for failure [44]. The load will be readjusted every 2 weeks with an interval of 2 to 4 days after the last treatment session to prevent residual exercise fatigue from interfering with the tests from 7 to $10 \mathrm{RM}$. As for the $1 \mathrm{RM}$ test, the 7-10 RM test has the advantage of minimizing the effect of pain on maximum strength generation [43].

The 7-10 RM estimation session and all sessions for all groups will start with 5 min of warm-up on a bicycle, before any intervention. The goal for the warm-up period is that participants exercise less than 11 (light) on Borg's perceived effort rating scale, scored from 6 to 20 [45]. For groups with exercises with load (BFR40\% + LL, BFR80\% + LL, and HIRE+BFRplacebo), strengthening will be performed through bilateral knee extension exercise (angle between $90^{\circ}$ and $45^{\circ}$ of knee flexion) on the chair extensor [46], based on previous protocols for protecting the patellofemoral joint during exercises [47]. The training parameters will be adjusted having the HIRE group as a reference, so that there is no difference in the training volume between groups with exercises, as shown in Table 1.

Participants will be warned that knee pain or discomfort during exercise is normal and that it does not necessarily cause joint damage [48]. The load will be reduced by $20 \%$ compared to the $1 \mathrm{RM}$ estimate if the pain prevents the volunteers from completing the exercise [49]. 


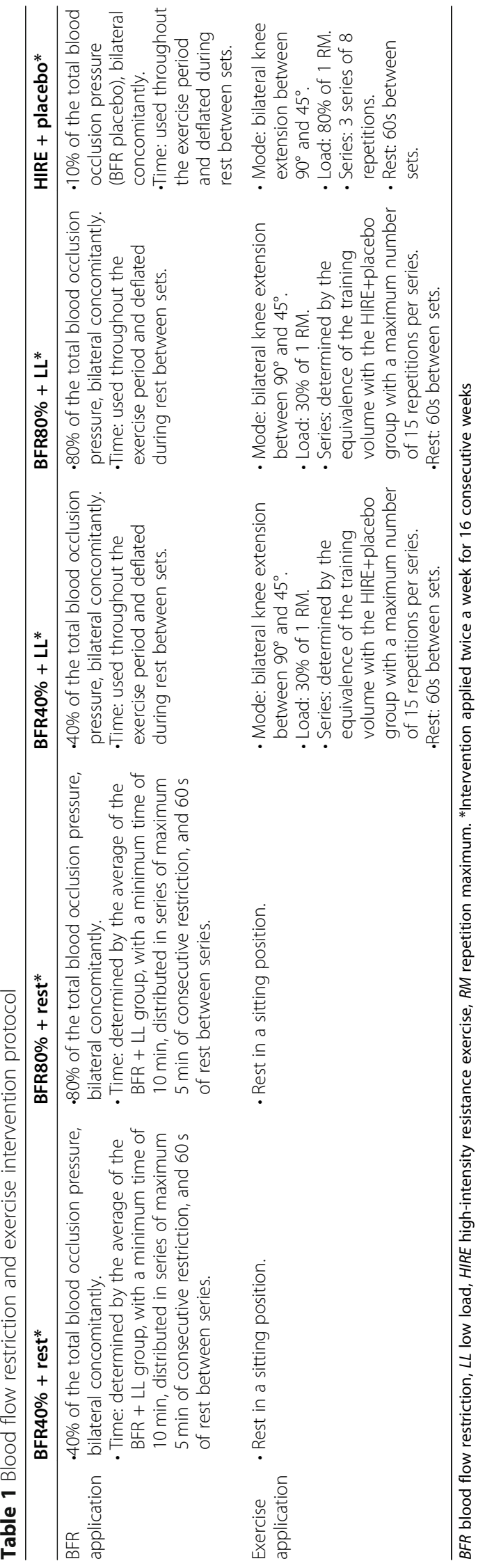




\section{Blood flow restriction}

The LOP will be estimated individually to generate a similar metabolic stimulus among the participants [42]. After 10 min of rest in a climatized room (between $23^{\circ}$ and $25^{\circ} \mathrm{C}$ ), the LOP will be determined with the volunteers seated and with the upper limbs relaxed at the side of the body. The transducer (5 to $10 \mathrm{MHZ}$ ) of a portable vascular Doppler (DV 610, Medmega) will be positioned at the ankle at a medium distance between the medial malleolus and the calcaneus tendon to capture the auscultatory signal from the posterior tibial artery [40]. A 13.5- $\mathrm{cm}$-wide pneumatic cuff will be positioned at the proximal end of the thigh and inflated until the auscultatory signal ceases, indicating occlusion of the artery [50, 51].

In the HIRE+placebo group, a minimum restriction pressure of $10 \%$ of the LOP will be applied, an extremely low dose that does not influence the volume of the exercises performed [52]. The same cuff used in the assessment of LOP will be inflated in the proximal end of the thigh with the participants seated for rest in the unloaded groups, and in knee extension exercises for the other groups. The cuff will be deflated during the rest interval between sets. Fluctuations in the prescribed pressure $(10 \%, 50 \%$, and $80 \%$ of LOP) will be monitored and regulated by the therapist. The number of repetitions completed in each series will be monitored to verify that the total proposed volume has been reached, then the cuff will be deflated.

During the evaluations and exercises, the position of the cuff will be adjusted so that the non-insufflated portion of the equipment is in the lateral region of the thigh, away from the femoral artery located in the medial compartment of the leg. This way, a smaller LOP is necessary [53], except for the HIRE+placebo group, in which the area without cuff insufflation will be positioned over the femoral artery so that there is the least possible blood restriction.

\section{Criteria for discontinuing or modifying allocated interventions}

The criteria for discontinuing or modifying intervention allocation of a particular participant from the study will be as follows: occurrence of severe adverse events or threat to life, as specified in the "Adverse event reporting and harms" section or if the participant has any health condition that forbids him from receiving study interventions.

If any participant discontinues the study or changes allocation, data will be analyzed considering the initial allocation of the participant as he/she was initially randomized. Once the interventions delivered (BFR and HIRE) are known to be safe [54], no interim analyses are planned for participants who discontinue before the study timeline, nor for external committees for data monitoring.

In addition, although the BFR and HIRE are known to be safe, adverse events, such as harm, could happen. If any participant gets harmed from interventions, the authors guarantee proper care by medical and/or physiotherapy professionals at the Federal University of Amapá.

\section{Plans to promote participant retention and complete follow-up}

For promoting participant retention and complete follow-up, the plans of the authors are as follows: make an understandable and as short as possible ICF; answer all questions from participants during ICF discussion and explain the importance of their participation, also clarifying and minimizing any concern from participants; ask about the participant's expectations and aligned them with the aim of the study; send reminders for upcoming visits and follow-up by mail and telephone numbers of participants and/or their closest family members; accommodate the schedule as much as possible; provide a comfortable and patient-friendly environment; and show participants authors' appreciation and recognition of their collaboration in the trial.

\section{Relevant concomitant care permitted or prohibited during the trial}

All subjects included will be instructed not to change the current dosage of their anti-inflammatory and analgesics and will be encouraged to report any medical or pharmacological interventions received during the study. In addition, participants will be discouraged to start regular exercises and any other treatment during the interventions of the study and should inform the researchers if these recommendations are broken. The volunteers will be reevaluated at the end of the 16 weeks of treatment, and then they will be instructed to practice the regular exercises of their choice.

\section{Masking/blinding}

The participants will not be informed about which group they belong to and will be instructed not to talk about their experience during the exercise in case they accidentally meet other participants. In addition to the placebo for the HIRE group, all participants will be informed that BFR is effective in increasing muscle strength and reducing knee pain.

The interventions, evaluations, randomization, and data statistical analyses will be carried out by different collaborators who will not share information about the research, making it difficult to influence the data assessed in different phases of the study. Despite these procedures, the characteristics of the interventions do 
not allow for blinding of participants, outcome assessors (self-reported), and therapists.

\section{Cognitive state}

The assessment of cognitive status will be carried out through the Mini-Mental State Examination (MMSE) [55], an instrument used for tracking dementia and assessing cognitive function [56]. The translated and validated version in Brazil was proposed by Bertolucci et al. [57] and Almeida [58]. Scores range from 0 to 30 points, with the cut-off point for cognitive decline taking into account the respondent's level of education, corresponding to the score: $\leq 13$ points for illiterates, $\leq 18$ points for those with 1 to 11 years of schooling, and $\leq 26$ points for schooling over 11 years [57]. Possible cognitive decline will imply in participant exclusion.

\section{Primary outcome}

The Western Ontario McMaster University Osteoarthritis Index (WOMAC) pain score is the primary outcome. WOMAC is a disease-specific quality of life questionnaire for use in osteoarthritis clinical trials [59]. The WOMAC pain subscale has 5 items, with the rating scale ranging from 0 (none) to 4 (extreme), with higher scores indicating worse painful conditions [60].

\section{Secondary outcomes}

\section{Evaluation of the severity of osteoarthritis}

The Lequesne Algofunctional Index and the WOMAC are commonly used instruments to assess pain, physical disability, and disease severity in patients diagnosed with OA [61]. Studies previously conducted show that WOMAC and the Lequesne Functional Something Index have a good correlation. Bellamy et al. [60] demonstrated that the questionnaires show similar results when used to record improvement of patients with OA submitted to medication.

According to Samuel and Kanimozhi [59] when analyzing the results used in the diagnosis, prognosis, and rehabilitation of patients with knee OA, the WOMAC, and the Lequesne Algofunctional Index in the pain subscale shows reliability and a good correlation of results. However, although both address the severity of OA and the physical function of these patients, the questionnaires are not a substitute for assessing the symptoms and disabilities of these cases [62].

\section{Functional assessment and risk of falls}

The Time Get Up and Go Test (TUG), 30-second Chair Stand Test (30-sCST), and 40-m Fast Paced Walk Test (40mFPWT) are tests recommended by the Osteoarthritis Research Society International (OARSI), considered as a basic set of functional tests for assessing physical performance in patients with knee OA [63]. All three tests (TUG, 30-sCST, and 40mFPWT) are also used to assess balance and risk of falls [64, 65], mainly due to their having good to excellent correlation with poor balance and number of falls $[66,67]$. The tests will be applied according to the protocol described by OARSI [63]. For TUG and 40mFPWT, tests completed in less time are associated with better fitness and lower risk of falling $[64,66]$. For 30-sCST, a higher number of repetitions indicates better functional prognosis [67].

\section{Clinical Test of Sensory Interaction and Balance Modified}

The Clinical Test of Sensory Interaction and Balance Modified test is a balance test that assesses the visual, vestibular, and somatosensory influence of static balance in six sensory conditions: (1) standing, standing on a stable surface, eyes open; (2) on a stable surface, standing still, with eyes closed; (3) on an unstable surface, standing, standing with eyes open; (4) standing, standing on an unstable surface with eyes closed. Individuals will be instructed to remain for $30 \mathrm{~s}$ in each sensory condition, without taking any steps to compensate for the instability, and without moving the upper limbs, heels, and feet; if instability occurs before $30 \mathrm{~s}$, the test is completed and considered as altered [68].

\section{Level of physical activity}

The level of physical activity will be verified using the Brazilian version of the International Physical Activity Questionnaire (IPAQ), long version. This questionnaire includes 27 questions about physical activities performed in a regular week, with vigorous, moderate, and light intensity, with a minimum duration of 10 continuous minutes, distributed in five domains: work, transportation, domestic activity, leisure/recreation and sitting. The level of physical activity will be classified continuously by calculating the estimated energy expenditure in METs (metabolic equivalent) [69].

\section{Evaluation of muscle strength of knee extensors}

The muscle strength of the knee extensors will be performed using the MicroFET2 ${ }^{\circ}$ manual dynamometer (Hogan Scientific - USA), which is a reliable and valid portable device for the evaluation of the isometric muscle strength and power of lower limbs [70]. The procedures for collecting the isometric strength of knee extensors will follow the steps of an already published study [71].

\section{Quality of life assessment}

The Brazilian version of the generic questionnaire of quality of life RAND 36-Item Short-Form Survey (SF-36) Version (1.0) [72] will be applied, which evaluates 8 domains, namely: functional capacity, limitation due to physical aspects, pain, general health, vitality, social 
aspects, emotional aspects, and mental health. The questionnaire score is given by domain and ranges from 0 to 100 , in which the highest score reflects the best function.

\section{Pain immediately after training}

Knee joint pain during exercise will be assessed in all sessions (immediately after each set) by the pain VAS. Participants will be asked to report their knee pain score before starting interventions and immediately at the end of each session. The final score will be the difference between the first and second score.

\section{Global Perceived Effect scale}

For this research, the Global Perceived Effect Scale (GPE) scale was adapted to assess the patient perception of recovery from the first session and will be applied during all follow-up points. The guiding question was "Compared to the day you started the treatment, how do you describe your knee pain today?" It is a numerical scale of 11 points ( -5 to 5 ), with -5 being much worse; 0 being no change; and 5 being complete recovery. The higher the score, the better the recovery from the condition [73].

\section{Sample size calculation}

Sample size is based on detecting non-inferiority of the groups that will receive interventions with BFR compared to active placebo with exercise. For the change in pain on the WOMAC subscale (score 0-20), a noninferiority margin (NIM) of 2.1 units was chosen as it is less than the minimal clinically important difference (MCID) reported as 2.4 points in the WOMAC questionnaire [74]. The margin of 2.1 units is the same of $11 \%$ of the total WOMAC pain subscale score, which is higher than the $8-9 \%$ commonly used in previous studies [75-77].

Assuming a standard deviation (SD) of 3.14 [78], 80\% power at an alpha level of 5\% to the primary measuring point $72 \mathrm{~h}$ after completion of interventions and a dropout of $15 \%$, we will need 33 participants per group, resulting in a total sample of 165 participants. The use of bilateral alpha value is in accordance with the recommendations of not ignoring superiority in non-inferiority trials $[79,80]$. The sample size calculation was performed using the R software version 4.1.2.

\section{Statistical analysis}

The statistical analysis will be conducted following the principles of intention-to-treat analysis. The normality distributions of the data will be assessed by visual inspection of histograms. In order to ensure data quality and data consistency between the source data and the data entered into the database, the data will be entered using the double entry method by two researchers (TSS and WNNS) independently into the database.

A linear mixed-effects model will be used to examine the between-group differences (BFR40\% + rest, BFR $80 \%$ + rest, BFR40\% + LL, BFR $80 \%$ + LL, and HIRE+placebo), time (baseline, $72 \mathrm{~h}, 20,28,40,52$, and 64 weeks), and group-time interaction in the primary outcome of pain intensity (measured using WOMAC subscale), and secondary outcomes of the disease severity, physical functional data, balance data, quality of life, pain during intervention, global perceived effect scale, and muscle strength. The 72-h follow-up will be the primary measurement point, and the other timepoints will be used to support the understanding of the effects of the interventions. Non-inferiority will be demonstrated if the lower limit of the $95 \%$ bilateral confidence intervals (CI) for the difference between the HIRE and each BFR group is above -2.1 units for change in the WOMAC pain subscale. The comparison between the BFRs groups and the secondary results will be interpreted using the superiority framework since we have not pre-defined any NIMs for these outcomes.

Analyses will be adjusted for baseline demographic and clinical characteristics of age, sex, disease severity, BMI, and educational level, considering $80 \%$ power at an alpha level of 5\%. The effect sizes (Cohen's $d$ ) of the analyses will be presented with their respective 95\% CIs. Chi-square tests will be used to test for possible differences in the adverse events observed between groups. All analyses will be calculated by one of the authors (NCRI) blinded to the allocation of groups, using tables with randomization codes. The statistical analysis will be conducted using the SPSS 25.0 software (SPSS, Chicago, IL, USA).

\section{Adverse event reporting and harms}

During the trial, we will collect and record possible adverse events, describing the date of onset and date of resolution, evaluating event severity, investigating potential causal relationships with the intervention or with other suspect drugs, and assessing the potential effect of the event.

Some adverse events that may occur with BFR include rhabdomyolysis, subcutaneous hemorrhage, numbness, cold feeling, deep vein thrombosis, and itching [81, 82] . Severity must be defined according to the following criteria:

- Mild: Awareness of signs or symptoms, but easily tolerable;

- Moderate: enough discomfort to interfere with normal daily activities;

- Severe: inability to perform normal daily activities; 
- Threat to life: immediate risk of death due to the reaction that occurred;

All adverse events will be tracked until the incident is resolved or the trial is completed.

\section{Discussion}

This trial will investigate whether BFR at different pressure levels isolated or combined with low-intensity exercise shows similar results to HIRE in pain for individuals with knee OA. In addition, the disease severity, muscle performance, physical function, risk of falling, and quality of life will also be assessed.

Although previous studies have shown pain reduction with BFR training in individuals with OA, the protocol of BFR application is very heterogeneous. Haphazardly, BFR is estimated at $70 \%$ of the occlusion pressure [28], $200 \mathrm{mmHg}$ [40], or through formulas [83]. In addition, its use is commonly combined with low-intensity resistance exercises. Then, the real effect of BFR in an elderly with chronic disease is unknown.

With this trial determining the best application of BFR, patients with knee OA, who do not adhere to traditional treatment protocols with physical exercise, will experience a new and less uncomfortable modality [40, 83]. If BFR proves to be an effective alternative for the treatment of knee OA, resources used for surgeries and care resulting from the worsening of the disease severity could be avoided every year.

In order to answer what is proposed, this trial uses a robust methodology because it is randomized, with hidden allocation, blinded statistical analyst, and used the intention-to-treat approach. Thus, it reduces the influence of selection bias, measurement bias, and other errors in the trial results.

However, this study has limitations. Due to the different nature of the interventions, it will not be possible to blind participants, outcome assessors (self-reported), and therapists. In addition, the measurement of the isometric strength muscle outcome can be influenced by the participant's learning effect during the test. Finally, the BFR will be determined at rest, and therefore, it will not be possible to guarantee that the amount of restricted blood flow is equal during the exercises, as the hemodynamics is altered due to muscle contraction and the release of substances that act on the blood vessels [84].

\section{Trial status}

The expected start of recruitment of participants is March 2022. Recruitment is expected to continue until March 2023, with a 1-year follow-up to be completed in July 2024. The data analysis is expected to be completed in December 2024.
The authors confirm that all ongoing and related trials for this drug/intervention are registered.

\begin{abstract}
Abbreviations
OA: Osteoarthritis; BFR: Blood flow restriction; BFRplacebo: Blood flow restriction placebo; LOP: Arterial limb occlusion pressure; BFR40\%: $40 \%$ of the total arterial limb occlusion pressure; BFR80\%: $80 \%$ of the total arterial limb occlusion pressure; HIRE: High-intensity resistance exercise; LL: Low load; RM: Repetition maximum; ICF: Informed Consent Form; VAS: Visual analog scale; MMSE: Mini-Mental State Exam; WOMAC: Western Ontario and McMaster Universities Osteoarthritis Index; OARSI: Osteoarthritis Research Society International; TUG: Time Get Up and Go Test; IPAQ: International Physical Activity Questionnaire; SF 36: Short-Form Health Survey; GPE: Global Perceived Effect
\end{abstract}

\section{Supplementary Information}

The online version contains supplementary material available at https://doi. org/10.1186/s13063-022-05998-3.

Additional file 1. SPIRIT Checklist. SPIRIT checklist completed.

\section{Authors' contributions}

RACJ, TSS, and WNNS contributed to the design of the project and writing of the manuscript. APM and NCRI contributed to the project design, critical review, and final approval of the manuscript. All authors read and approved the final manuscript.

\section{Funding}

The Federal University of Amapá is the institution responsible for the trial, enabling the logistical structure and volunteers. However, there is no type of direct monetary financing for the study, which will be financed with the authors' own resources.

\section{Availability of data and materials}

Datasets generated or analyzed during the current study will be openly available in a repository.

\section{Declarations}

\section{Ethics approval and consent to participate}

The study was approved by the Research Ethical Committee of the Federal University of Amapá under the protocol number 4.248.398. Personal information about potential and enrolled participants will be protected and strictly confidential before, during, and after the trial. This confidentiality will be ensured through numerical codes to identify the participants and controlled access by the authors to the database and forms of the participants. Data of the results without personal information from this study will be planned to disseminate in conferences or peer-reviewed publications. All changes to the study protocol will be registered in the Clinical Trials Brazil (http://www.ensaiosclinicos.gov.br) under number RBR-93rx9q and sent to the local Research Ethical Committee.

\section{Consent for publication}

Not applicable.

\section{Competing interests}

The authors declare that they have no competing interests.

\section{Author details}

${ }^{1}$ Postgraduate Program of Health Sciences, Department of Biological Sciences and Health, Federal University of Amapá - UNIFAP, Macapá, AP, Brazil. ${ }^{2}$ Physical Therapy Course, Department of Biological Sciences and Health, Federal University of Amapá - UNIFAP, Macapá, AP, Brazil. 
Received: 14 January 2021 Accepted: 3 January 2022

\section{Published online: 15 January 2022}

\section{References}

1. Hunter DJ, Bierma-Zeinstra S. Osteoarthritis. Lancet. 2019;393(10182):174559. https://doi.org/10.1016/S0140-6736(19)30417-9.

2. O'Neill TW, McCabe PS, McBeth J. Update on the epidemiology, risk factors and disease outcomes of osteoarthritis. Best Pract Res Clin Rheumatol. 2018; 32(2):312-26. https://doi.org/10.1016/j.berh.2018.10.007.

3. James SL, Abate D, Abate KH, Abay SM, Abbafati C, Abbasi N, et al. Global, regional, and national incidence, prevalence, and years lived with disability for 354 diseases and injuries for 195 countries and territories, 1990-2017: a systematic analysis for the Global Burden of Disease Study 2017. Lancet. 2018;392(10159):1789-858. https://doi.org/10.1016/S0140-6736(18)32279-7.

4. Hunter DJ, Schofield D, Callander E. The individual and socioeconomic impact of osteoarthritis. Nat Rev Rheumatol. 2014;10(7):437-41. https://doi. org/10.1038/nrrheum.2014.44

5. Fransen M, McConnell S, Harmer AR, der Esch MV, Simic M, Bennell KL. Exercise for osteoarthritis of the knee: a Cochrane systematic review. $\mathrm{Br}$ J Sports Med. 2015;49:1554-7. [cited 2020 Mar 10]; Available from: https:// www.cochranelibrary.com/cdsr/doi/10.1002/14651858.CD004376.pub3/full.

6. Issa SN, Sharma L. Epidemiology of osteoarthritis: an update. Curr Rheumatol Rep. 2006;8(1):7-15. https://doi.org/10.1007/s11926-006-0019-1.

7. Muraki S, Akune T, Teraguchi M, Kagotani R, Asai Y, Yoshida M, et al. Quadriceps muscle strength, radiographic knee osteoarthritis and knee pain: the ROAD study. BMC Musculoskelet Disord [Internet]. 2015;16:305 Available from: https://www.ncbi.n/m.nih.gov/pmc/articles/PMC4609096/.

8. Gallegly JC, Turesky NA, Strotman BA, Gurley CM, Peterson CA, DupontVersteegden EE. Satellite cell regulation of muscle mass is altered at old age. J Appl Physiol. Am Physiol Soc. 2004;97(3):1082-90. https://doi.org/1 0.1152/japplphysiol.00006.2004.

9. Mobasheri A, Matta C, Zákány R, Musumeci G. Chondrosenescence: definition, hallmarks and potential role in the pathogenesis of osteoarthritis. Maturitas. 2015;80(3):237-44. https://doi.org/10.1016/j.maturitas.2014.12.003.

10. Anderson AS, Loeser RF. Why is osteoarthritis an age-related disease? Best Pract Res Clin Rheumatol. 2010;24(1):15-26. https://doi.org/10.1016/j.berh.2 009.08.006.

11. Harvey JA, Chastin SFM, Skelton DA. Prevalence of sedentary behavior in older adults: a systematic review. Int J Environ Res Public Health. 2013; 10(12):6645-61. https://doi.org/10.3390/ijerph10126645.

12. Bannuru RR, Osani MC, Vaysbrot EE, Arden NK, Bennell K, Bierma-Zeinstra SMA, et al. OARSI guidelines for the non-surgical management of knee, hip, and polyarticular osteoarthritis. Osteoarthritis Cartilage. 2019;27(11):1578-89. https://doi.org/10.1016/j.joca.2019.06.011.

13. Borde R, Hortobágyi T, Granacher U. Dose-response relationships of resistance training in healthy old adults: a systematic review and metaanalysis. Sports Med. 2015;45(12):1693-720. https://doi.org/10.1007/s40279015-0385-9.

14. Brosseau L, Taki J, Desjardins B, Thevenot O, Fransen M, Wells GA, et al. The Ottawa panel clinical practice guidelines for the management of knee osteoarthritis. Part two: strengthening exercise programs. Clin Rehabil. 2017; 31(5):596-611. https://doi.org/10.1177/0269215517691084.

15. Kolasinski SL, Neogi T, Hochberg MC, Oatis C, Guyatt G, Block J, et al. 2019 American College of Rheumatology/Arthritis Foundation Guideline for the Management of Osteoarthritis of the Hand, Hip, and Knee. Arthritis Care Res. 2020;72(2):149-62. https://doi.org/10.1002/acr.24131.

16. Teo PL, Hinman RS, Egerton T, Dziedzic KS, Bennell KL. Identifying and prioritizing clinical guideline recommendations most relevant to physical therapy practice for hip and/or knee osteoarthritis. J Orthop Sports Phys Ther. 2019;49(7):501-12. https://doi.org/10.2519/jospt.2019.8676.

17. World Health Organization. Global Recommendations on Physical Activity for Health [Internet]. Geneva: World Health Organization; 2010 [cited 2020 Apr 11]. Available from: http://www.ncbi.n/m.nih.gov/books/NBK305057/

18. Wallis JA, Webster KE, Levinger P, Taylor NF. What proportion of people with hip and knee osteoarthritis meet physical activity guidelines? A systematic review and meta-analysis. Osteoarthr Cartil. 2013;21(11):1648-59. https://doi.org/10.1016/j.joca.2013.08.003.

19. Jan M-H, Lin J-J, Liau J-J, Lin Y-F, Lin D-H. Investigation of clinical effects of high- and low-resistance training for patients with knee osteoarthritis: a randomized controlled trial. Phys Ther. 2008;88(4):427-36. https://doi.org/1 0.2522/ptj.20060300.
20. Bennell KL, Dobson F, Hinman RS. Exercise in osteoarthritis: moving from prescription to adherence. Best Pract Res Clin Rheumatol. 2014;28(1):93-117. https://doi.org/10.1016/j.berh.2014.01.009.

21. Bartels EM, Juhl CB, Christensen R, Hagen KB, Danneskiold-Samsøe B, Dagfinrud $\mathrm{H}$, et al. Aquatic exercise for the treatment of knee and hip osteoarthritis. Cochrane Database Syst Rev. 2016;3:CD005523. https://doi. org/10.1002/14651858.CD005523.pub3.

22. Health Quality Ontario. Structured education and neuromuscular exercise program for hip and/or knee osteoarthritis: a health technology assessment. Ont Health Technol Assess Ser. 2018;18:1-110.

23. Pazit L, Jeremy D, Nancy B, Michael B, George E, Hill KD. Safety and feasibility of high speed resistance training with and without balance exercises for knee osteoarthritis: a pilot randomised controlled trial. Phys Ther Sport Off J Assoc Chart Physiother Sports Med. 2018;34:154-63. https:// doi.org/10.1016/j.ptsp.2018.10.001.

24. Patterson SD, Hughes L, Warmington S, Burr J, Scott BR, Owens J, et al. Blood flow restriction exercise: considerations of methodology, application, and safety. Front Physiol [Internet]. 2019;10:533 Available from: https://www. ncbi.nlm.nih.gov/pmc/articles/PMC6530612/.

25. Hughes L, Paton B, Rosenblatt B, Gissane C, Patterson SD. Blood flow restriction training in clinical musculoskeletal rehabilitation: a systematic review and meta-analysis. Br J Sports Med. 2017;51(13):1003-11. https://doi. org/10.1136/bjsports-2016-097071.

26. Conceição MS, Gáspari AF, Ramkrapes APB, Junior EMM, Bertuzzi R, Cavaglieri $C R$, et al. Anaerobic metabolism induces greater total energy expenditure during exercise with blood flow restriction. PloS One. 2018; 13(3):e0194776. https://doi.org/10.1371/journal.pone.0194776.

27. Loenneke JP, Kim D, Fahs CA, Thiebaud RS, Abe T, Larson RD, et al. Effects of exercise with and without different degrees of blood flow restriction on torque and muscle activation. Muscle Nerve. 2015;51(5):713-21. https://doi. org/10.1002/mus.24448.

28. Bryk FF, Dos Reis AC, Fingerhut D, Araujo T, Schutzer M, Cury RDPL, et al. Exercises with partial vascular occlusion in patients with knee osteoarthritis: a randomized clinical trial. Knee Surg Sports Traumatol Arthrosc Off J ESSKA. 2016;24(5):1580-6. https://doi.org/10.1007/s00167-016-4064-7.

29. Segal NA, Williams GN, Davis MC, Wallace RB, Mikesky AE. Efficacy of blood flow-restricted, low-load resistance training in women with risk factors for symptomatic knee osteoarthritis. PM R. 2015;7(4):376-84. https://doi.org/10.1 016/j.pmrj.2014.09.014

30. Segal N, Davis MD, Mikesky AE. Efficacy of blood flow-restricted low-load resistance training for quadriceps strengthening in men at risk of symptomatic knee osteoarthritis. Geriatr Orthop Surg Rehabil. 2015;6(3):160 7. https://doi.org/10.1177/2151458515583088.

31. Lixandrão ME, Roschel H, Ugrinowitsch C, Miquelini M, Alvarez IF, Libardi CA. Blood-flow restriction resistance exercise promotes lower pain and ratings of perceived exertion compared with either high- or low-intensity resistance exercise performed to muscular failure. J Sport Rehabil. 2019; 28(7):706-10. https://doi.org/10.1123/jsr.2018-0030.

32. Abe T, Kearns CF, Sato Y. Muscle size and strength are increased following walk training with restricted venous blood flow from the leg muscle, Kaatsu-walk training. J Appl Physiol. 2006;100:1460-6.

33. Kang DY, Kim HS, Lee KS, Kim YM. The effects of bodyweight-based exercise with blood flow restriction on isokinetic knee muscular function and thigh circumference in college students. J Phys Ther Sci. 2015;27(9):2709-12. https://doi.org/10.1589/.jpts.27.2709.

34. Yasuda T, Loenneke JP, Thiebaud RS, Abe T. Effects of blood flow restricted low-intensity concentric or eccentric training on muscle size and strength. PloS One. 2012;7(12):e52843. https://doi.org/10.1371/journal.pone.0052843.

35. Murlasits Z, Reed J, Wells K. Effect of resistance training frequency on physiological adaptations in older adults. J Exerc Sci Fit. 2012;10(1):28-32. https://doi.org/10.1016/j.jesf.2012.04.006.

36. Shimizu R, Hotta K, Yamamoto S, Matsumoto T, Kamiya K, Kato M, et al. Low-intensity resistance training with blood flow restriction improves vascular endothelial function and peripheral blood circulation in healthy elderly people. Eur J Appl Physiol. 2016;116(4):749-57. https://doi.org/10.1 007/s00421-016-3328-8.

37. Counts BR, Dankel SJ, Barnett BE, Kim D, Mouser JG, Allen KM, et al. Influence of relative blood flow restriction pressure on muscle activation and muscle adaptation. Muscle Nerve. 2016;53(3):438-45. https://doi.org/1 $0.1002 /$ mus.24756. 
38. Chan A-W, Tetzlaff JM, Gøtzsche PC, Altman DG, Mann H, Berlin JA, et al. SPIRIT 2013 explanation and elaboration: guidance for protocols of clinical trials. BMJ [Internet]. 2013;346:e7586 Available from: https://www.bmj.com/ content/346/bmj.e7586.

39. Boutron IG, Altman D, Moher DF, Schulz K, Ravaud P. CONSORT Statement for Randomized Trials of Nonpharmacologic Treatments: a 2017 update and a CONSORT Extension for Nonpharmacologic Trial Abstracts. Ann Intern Med [Internet]. 2017;167:40-7. Available from: https://www.acpjournals.org/ doi/abs/10.7326/M17-0046

40. Ferraz RB, Gualano B, Rodrigues R, Kurimori CO, Fuller R, Lima FR, et al. Benefits of resistance training with blood flow restriction in knee osteoarthritis. Med Sci Sports Exerc. 2018;50(5):897-905. https://doi.org/1 0.1249/MSS.0000000000001530.

41. Crossley KW, Porter DA, Ellsworth J, Caldwell T, Feland JB, Mitchell U, et al. Effect of cuff pressure on blood flow during blood flow-restricted rest and exercise. Med Sci Sports Exerc. 2020;52(3):746-53. https://doi.org/10.1249/ MSS.0000000000002156.

42. Scott BR, Loenneke JP, Slattery KM, Dascombe BJ. Exercise with blood flow restriction: an updated evidence-based approach for enhanced muscular development. Sports Med Auckl NZ. 2015;45(3):313-25. https://doi.org/10.1 007/s40279-014-0288-1.

43. Abdul-Hameed U, Rangra P, Shareef Mohd Y, Hussain Mohd E. Reliability of 1-repetition maximum estimation for upper and lower body muscular strength measurement in untrained middle aged type 2 diabetic patients. Asian J Sports Med. 2012;3:267-73.

44. Nascimento MA do, Cyrino ES, Nakamura FY, Romanzini M, Pianca HJC, Queiróga MR. Validação da equação de Brzycki para a estimativa de 1-RM no exercício supino em banco horizontal. Rev Bras Med Esporte. Sociedade Brasileira de Medicina do Exercício e do Esporte. 2007;13:47-50.

45. Klonizakis M, Tew GA, Gumber A, Crank H, King B, Middleton G, et al. Supervised exercise training as an adjunct therapy for venous leg ulcers: a randomized controlled feasibility trial. Br J Dermatol. 2018;178(5):1072-82. https://doi.org/10.1111/bjd.16089.

46. Cook SB, LaRoche DP, Villa MR, Barile H, Manini TM. Blood flow restricted resistance training in older adults at risk of mobility limitations. Exp Gerontol. 2017;99:138-45. https://doi.org/10.1016/j.exger.2017.10.004.

47. Biscarini A. Minimization of the knee shear joint load in leg-extension equipment. Med Eng Phys. 2008;30(8):1032-41. https://doi.org/10.1016/j. medengphy.2007.12.012

48. Kanavaki AM, Rushton A, Efstathiou N, Alrushud A, Klocke R, Abhishek A, et al. Barriers and facilitators of physical activity in knee and hip osteoarthritis: a systematic review of qualitative evidence. BMJ Open. 2017; 7(12):e017042. https://doi.org/10.1136/bmjopen-2017-017042.

49. Levinger P, Dunn J, Bifera N, Butson M, Elias G, Hill KD. High-speed resistance training and balance training for people with knee osteoarthritis to reduce falls risk: study protocol for a pilot randomized controlled trial. Trials. 2017;18(1):384. https://doi.org/10.1186/s13063-017-2129-7.

50. Loenneke JP, Fahs CA, Rossow LM, Sherk VD, Thiebaud RS, Abe T, et al. Effects of cuff width on arterial occlusion: implications for blood flow restricted exercise. Eur J Appl Physiol. 2012;112(8):2903-12. https://doi.org/1 0.1007/s00421-011-2266-8

51. Weatherholt AM, Vanwye WR, Lohmann J, Owens JG. The effect of cuff width for determining limb occlusion pressure: a comparison of blood flow restriction devices. Int J Exerc Sci. 2019;12(3):136-43.

52. Mattocks KT, Jessee MB, Counts BR, Buckner SL, Grant Mouser J, Dankel SJ, et al. The effects of upper body exercise across different levels of blood flow restriction on arterial occlusion pressure and perceptual responses. Physiol Behav. 2017;171:181-6. https://doi.org/10.1016/j.physbeh.2017.01.015.

53. Spitz RW, Bell ZW, Wong V, Viana RB, Chatakondi RN, Abe T, et al. The position of the cuff bladder has a large impact on the pressure needed for blood flow restriction. Physiol Meas. 2020;41:01NT01.

54. Minniti MC, Statkevich AP, Kelly RL, Rigsby VP, Exline MM, Rhon DI, et al. The safety of blood flow restriction training as a therapeutic intervention for patients with musculoskeletal disorders: a systematic review. Am J Sports Med. 2020;48(7):1773-85. https://doi.org/10.1177/0363546519882652.

55. Folstein MF, Folstein SE, McHugh PR. "Mini-mental state". A practical method for grading the cognitive state of patients for the clinician. J Psychiatr Res. 1975;12(3):189-98. https://doi.org/10.1016/0022-3956(75)90026-6.

56. Lourenço RA, Veras RP. Mini-Exame do Estado Mental: características psicométricas em idosos ambulatoriais. Rev Saúde Pública. Faculdade de
Saúde Pública da Universidade de São Paulo. 2006;40(4):712-9. https://doi org/10.1590/S0034-89102006000500023.

57. Bertolucci PHF, Brucki SMD, Campacci SR, Juliano Y. O Mini-Exame do Estado Mental em uma população geral: impacto da escolaridade. Arq Neuropsiquiatr. Associação Arquivos de Neuro-Psiquiatria. 1994;52(1):1-7. https://doi.org/10.1590/S0004-282X1994000100001.

58. Almeida OP. Mini exame dos estado mental e o diagnóstico de demência no Brasil. Arq Neuropsiquiatr. Associação Arquivos de Neuro-Psiquiatria. 1998;56(3B):605-12. https://doi.org/10.1590/S0004-282X1998000400014.

59. Samuel AJ, Kanimozhi D. Outcome measures used in patient with knee osteoarthritis: With special importance on functional outcome measures. Int J Health Sci. 2019;13(1):52-60.

60. Bellamy N, Kean WF, Buchanan WW, Gerecz-Simon E, Campbell J. Double blind randomized controlled trial of sodium meclofenamate (Meclomen) and diclofenac sodium (Voltaren): post validation reapplication of the WOMAC Osteoarthritis Index. J Rheumatol. 1992;19:153-9.

61. White DK, Master H. Patient-reported measures of physical function in knee osteoarthritis. Rheum Dis Clin N Am. 2016;42(2):239-52. https://doi.org/10.1 016/j.rdc.2016.01.005.

62. Georgiev T. Clinical characteristics and disability in patients with knee osteoarthritis: real world experience from Bulgaria. Reumatologia. 2019;57(2): 78-84. https://doi.org/10.5114/reum.2019.84812.

63. Dobson F, Hinman RS, Roos EM, Abbott JH, Stratford P, Davis AM, et al. OARSI recommended performance-based tests to assess physical function in people diagnosed with hip or knee osteoarthritis. Osteoarthr Cartil. 2013; 21(8):1042-52. https://doi.org/10.1016/j.joca.2013.05.002.

64. Beauchet O, Fantino B, Allali G, Muir SW, Montero-Odasso M, Annweiler C. Timed Up and Go test and risk of falls in older adults: a systematic review. J Nutr Health Aging. 2011;15(10):933-8. https://doi.org/10.1007/s12603-0110062-0.

65. Mat S, Ng CT, Tan PJ, Ramli N, Fadzli F, Rozalli Fl, et al. Effect of modified Otago Exercises on postural balance, fear of falling, and fall risk in older fallers with knee osteoarthritis and impaired gait and balance: a secondary analysis. PM R. 2018;10(3):254-62. https://doi.org/10.1016/j.pmrj.2017.08.405.

66. Bennell K, Dobson F, Hinman R. Measures of physical performance assessments: Self-Paced Walk Test (SPWT), Stair Climb Test (SCT), Six-Minute Walk Test (6MWT), Chair Stand Test (CST), Timed Up \& Go (TUG), Sock Test, Lift and Carry Test (LCT), and Car Task. Arthritis Care Res. 2011;63(Suppl 11): S350-70. https://doi.org/10.1002/acr.20538.

67. Roongbenjawan N, Siriphorn A. Accuracy of modified 30-s chair-stand test for predicting falls in older adults. Ann Phys Rehabil Med. 2020;63(4):309-15 https://doi.org/10.1016/j.rehab.2019.08.003.

68. Hagedorn DK, Holm E. Effects of traditional physical training and visual computer feedback training in frail elderly patients. A randomized intervention study. Eur J Phys Rehabil Med. 2010;46(2):159-68.

69. Matsudo S, Araújo T, Matsudo V, Andrade D, Andrade E, Oliveira LC, et al. QUESTIONÁRIO INTERNACIONAL DE ATIVIDADE FÍSICA (IPAQ): ESTUPO DE VALIDADE E REPRODUTIBILIDADE NO BRASIL. Rev Bras Atividade Física Saúde. 2001;6:5-18.

70. Mentiplay BF, Perraton LG, Bower KJ, Adair B, Pua Y-H, Williams GP, et al. Assessment of lower limb muscle strength and power using hand-held and fixed dynamometry: a reliability and validity study. PLoS One [Internet]. 2015;10:e0140822 Available from: https://www.ncbi.nlm.nih.gov/pmc/a rticles/PMC4624940/.

71. Suzuki $Y$, lijima H, Tashiro $Y$, Kajiwara $Y$, Zeidan H, Shimoura K, et al. Home exercise therapy to improve muscle strength and joint flexibility effectively treats pre-radiographic knee OA in community-dwelling elderly: a randomized controlled trial. Clin Rheumatol. 2019;38(1):133-41. https://doi. org/10.1007/s10067-018-4263-3.

72. Ciconelli RM, Ferraz MB, Santos W, Meinão I, Quaresma MR. Tradução para a língua portuguesa e validação do questionário genérico de avaliação de qualidade de vida SF-36 (Brasil SF-36). Rev Bras Reum. 1999;39:143-50.

73. Freitas P, Pires D, Nunes C, Cruz EB. Cross-cultural adaptation and psychometric properties of the European Portuguese version of the Global Perceived Effect Scale in patients with chronic low back pain. Disabil Rehabil. 2019:43(7):1-7. https://doi.org/10.1080/09638288.2019.1648568.

74. Angst F, Aeschlimann A, Stucki G. Smallest detectable and minimal clinically important differences of rehabilitation intervention with their implications for required sample sizes using WOMAC and SF-36 quality of life measurement instruments in patients with osteoarthritis of the lower 
extremities. Arthritis Rheum. 2001;45(4):384-91. https://doi.org/10.1002/152 9-0131(200108)45:4<384:.AID-ART352>3.0.CO;2-0.

75. Berenbaum F, Grifka J, Cazzaniga S, D'Amato M, Giacovelli G, Chevalier X, et al. A randomised, double-blind, controlled trial comparing two intraarticular hyaluronic acid preparations differing by their molecular weight in symptomatic knee osteoarthritis. Ann Rheum Dis. 2012;71(9):1454-60. https://doi.org/10.1136/annrheumdis-2011-200972.

76. Hochberg MC, Martel-Pelletier J, Monfort J, Möller I, Castillo JR, Arden N, et al. Combined chondroitin sulfate and glucosamine for painful knee osteoarthritis: a multicentre, randomised, double-blind, non-inferiority trial versus celecoxib. Ann Rheum Dis. 2016;75(1):37-44. https://doi.org/10.113 6/annrheumdis-2014-206792.

77. Schnitzer TJ, Kivitz A, Frayssinet H, Duquesroix B. Efficacy and safety of naproxcinod in the treatment of patients with osteoarthritis of the knee: a 13-week prospective, randomized, multicenter study. Osteoarthr Cartil. 2010; 18(5):629-39. https://doi.org/10.1016/j.joca.2009.12.013.

78. Allen KD, Arbeeva L, Callahan LF, Golightly YM, Goode AP, Heiderscheit BC et al. Physical therapy vs internet-based exercise training for patients with knee osteoarthritis: results of a randomized controlled trial. Osteoarthr Cartil. 2018;26(3):383-96. https://doi.org/10.1016/j.joca.2017.12.008.

79. Dunn DT, Copas AJ, Brocklehurst P. Superiority and non-inferiority: two sides of the same coin? Trials. 2018;19(1):499. https://doi.org/10.1186/s13063-01 8-2885-z.

80. Committee for Proprietary Medicinal Products. Points to consider on switching between superiority and non-inferiority. Br J Clin Pharmacol. 2001; 52:223-8.

81. Iversen E, Røstad V. Low-load ischemic exercise-induced rhabdomyolysis. Clin J Sport Med Off J Can Acad Sport Med. 2010;20(3):218-9. https://doi. org/10.1097/JSM.0b013e3181df8d10.

82. Nakajima T, Kurano $\mathrm{M}$, lida $\mathrm{H}$, Takano $\mathrm{H}$, Oonuma $\mathrm{H}$, Morita $\mathrm{T}$, et al. Use and safety of KAATSU training: results of a national survey. Int J KAATSU Train Res. 2006;2(1):5-13. https://doi.org/10.3806/ijktr.2.5.

83. Harper SA, Roberts LM, Layne AS, Jaeger BC, Gardner AK, Sibille KT, et al. Blood-flow restriction resistance exercise for older adults with knee osteoarthritis: a pilot randomized clinical trial. J Clin Med. 2019;8(2). https:// doi.org/10.3390/jcm8020265.

84. Barbosa JB, Maia TO, Alves PS, Bezerra SD, Moura EC, Medeiros AlC, et al. Does blood flow restriction training increase the diameter of forearm vessels in chronic kidney disease patients? A randomized clinical trial. J Vasc Access. 2018;19(6):626-33. https://doi.org/10.1177/1129729818768179.

\section{Publisher's Note}

Springer Nature remains neutral with regard to jurisdictional claims in published maps and institutional affiliations.

Ready to submit your research? Choose BMC and benefit from:

- fast, convenient online submission

- thorough peer review by experienced researchers in your field

- rapid publication on acceptance

- support for research data, including large and complex data types

- gold Open Access which fosters wider collaboration and increased citations

- maximum visibility for your research: over $100 \mathrm{M}$ website views per year

At BMC, research is always in progress.

Learn more biomedcentral.com/submissions 